\title{
Perihelion Evolution of Observed New Comets Implies the Dominance of the Galactic Tide in Making Oort Cloud Comets Discernable
}

\author{
John J. Matese ${ }^{1}$ \\ Jack J. Lissauer ${ }^{2}$ \\ ${ }^{1}$ Department of Physics, University of Louisiana at Lafayette \\ Lafayette, LA, 70504-4210 \\ ${ }^{2}$ Space Science Division, MS 245-3, NASA Ames Research Center \\ Moffett Field, CA, 94035
}

1 Tel: (337) 482 6697; Fax: (337) 482 6699; E-mail: matese@louisiana.edu

Published 2004 Icarus 170, 508-513. 
Abstract: For an Oort cloud comet to be seen as a new comet, its perihelion must be moved from a point exterior to the loss cylinder boundary to a point interior to observable limits in a single orbit. The galactic tide can do this continuously, in a non-impulsive manner. Near-parabolic comets, with specific angular momentum $H \propto \sqrt{q}$, will most easily be made observable. Therefore, to reduce the perihelion distance, $H$ must decrease. Since weakly perturbed comets are, in general, more numerous than strongly perturbed comets, we can anticipate that new comets made observable by a weak tidal torque will more likely be first observed when their slowly changing perihelion distances are approaching their minimum osculating values under the action of the tide, rather than receding from their minimum values. That is, defining $\Delta \mathbf{H}_{\text {tide }}$ as the vector change due to the galactic tidal torque during the prior orbit, and $\mathbf{H}_{o b s}$ as the observed vector, the $\operatorname{sign} S \equiv \operatorname{Sign}\left(\mathbf{H}_{o b s} \cdot \Delta \mathbf{H}_{\text {tide }}\right)$ will more likely be -1 than +1 if a weak galactic tidal perturbation indeed dominates in making comets observable. Using comet data of the highest quality class (1A) for new comets ( $a>10,000 \mathrm{AU})$, we find that 49 comets have $S=-1$ and 22 have $S=+1$. The binomial probability that as many or more would exhibit this characteristic if in fact $S=\mp 1$ were equally likely is only 0.0009 . This characteristic also persists in other long-period comet populations, lending support to the notion that they are dominated by comets recently arrived from the outer Oort Cloud. The preponderance of $S=-1$ also correlates with weakly perturbed (i.e., smaller semimajor axis) new comets in a statistically significant manner. This is strong evidence that the data are of sufficiently high quality and sufficiently free of observational selection effects to detect this unique imprint of the tide.

Key Words: comets, dynamics; celestial mechanics. 


\section{INTRODUCTION}

The outer Oort cloud is formally defined as the ensemble of comets having original semimajor axes $a \geq 10^{4} \mathrm{AU}$ (Oort 1950). It has been shown that the vast majority of these comets are first-time entrants into the inner planetary region (Fernandez 1981), and these comets are therefore commonly referred to as new. The dominance of the galactic tide in making Oort cloud comets observable has been established on theoretical grounds (Heisler and Tremaine 1986). In contrast, the observational evidence for this dominance has not been compelling (Delsemme 1987, Matese and Whitman 1992, Wiegert and Tremaine 1999).

We present here a discussion of a subtle characteristic of the galactic tidal dominance which is difficult to mimic with observational selection effects or bad data. The statistical significance of our conclusions exceed anything found in prior comparisons of theoretical and observational orbital element distributions and constitute the strongest evidence yet that the galactic tide dominates stellar impulses in making new Oort cloud comets observable. Further, we find evidence that this statistic maintains a high level of significance in the young $\left(10^{4} \mathrm{AU} \geq a \geq 10^{3} \mathrm{AU}\right)$ generation and the old ( $\left.10^{3} \mathrm{AU} \geq a \geq 40 \mathrm{AU}\right)$ generation, though reduced from that found in the new family. This provides strong support that the young and old generations of long-period comets are indeed dominated by returning daughters from the new family first made observable by the tide.

Our dynamical analysis of the effects of the galactic tidal forces is presented in Section 2. Section 3 gives our results. In Section 4, we summarize our predictions and the observations and present our conclusions.

\section{DYNAMICS OF THE GALACTIC TIDE}

The dynamics of the galactic tide acting on near-parabolic Oort cloud comets is most simply given in a Newtonian framework (Matese and Whitmire 1996, Matese and Lissauer 2002). It describes how the angular momentum and the perihelion distance $\left(H \approx \sqrt{2 \mathrm{GM}_{\odot} q}, \mathbf{H} \perp \mathbf{q}\right)$ are changed by the galactic 
tide. Also of interest is the evolution of the major axis orientation, which we describe in terms of the Laplace-Runge-Lenz vector, e, that is directed toward the osculating aphelion point

$$
\mathbf{e}=e \widehat{Q} \equiv \widehat{R}-\frac{\dot{\mathbf{R}} \times \mathbf{H}}{{\sqrt{\mathrm{GM}_{\odot}}}_{\odot}}=e(\cos B \cos L, \cos B \sin L, \sin B)
$$

We denote the original value of semimajor axis, prior to perturbation by the planets, as $a$. Our choice of the remaining five independent orbital elements includes the time of passage of perihelion, $\tau$, the aphelion latitude, $B$, and longitude, $L$, the magnitude of the comet's specific angular momentum, $H$, and its orientation angle about the major axis, $\alpha$, which is defined in Eq. 3 below.

Near-parabolic comets are most likely to have their perihelia reduced to the observable region. Obtaining the secular change in angular momentum over an orbital period, $P_{a}$, we have

$$
\Delta \mathbf{H}_{\text {tide }}=\langle\dot{\mathbf{H}}\rangle P_{a} \approx \frac{5}{2} P_{a} a^{2} \Omega_{z}{ }^{2} \cos B(\hat{\phi} \sin B(1+\epsilon \cos 2 L)+\hat{\theta} \epsilon \sin 2 L)
$$

where $\hat{Q}, \hat{\phi} \equiv(\hat{z} \times \hat{Q}) / \cos B, \hat{\theta} \equiv(\hat{\phi} \times \hat{Q})$ are conventional spherical unit vectors, $\Omega_{\circ} \approx 2 \pi / 240$ Myr , $\Omega_{z} \approx 2 \pi / 90$ Myr and $\epsilon \equiv \Omega_{\circ}{ }^{2} / \Omega_{z}{ }^{2} \approx 0.1$ (Merrifield 1992, Holmberg and Flynn 2000). Both components of the vector time derivative $\dot{\mathbf{H}}$ are slowly varying. In contrast, because $\mathbf{H}$ itself is rapidly changing, any formalism which secularly averages the scalar time derivative

$$
\dot{H}=H^{-1}(\mathbf{H} \cdot \dot{\mathbf{H}})=H^{-1}\left(H_{\phi} \dot{H}_{\phi}+H_{\theta} \dot{H}_{\theta}\right) \equiv-\cos \alpha \dot{H}_{\phi}-\sin \alpha \dot{H}_{\theta}
$$

(as in Heisler and Tremaine 1986) will find $\dot{H}$ to be rapidly varying since $\alpha$, the orientation angle of $\mathbf{H}$ about the major axis, is rapidly changing.

In the loss cylinder model, any long-period comet that has a perihelion distance interior to the loss cylinder boundary will likely have experienced a planetary impulse that changed its energy sufficiently to have removed the comet from the new family. The comet is either ejected or turned into a daughter member of the young or old generations (Fernandez 1981). Therefore, for new comets to be recognized as coming from the outer Oort cloud on their prior orbit, they must undergo galactic tidal changes in the angular momentum from their prior value as they receded from the planetary region to their observed value as they 
return interior to the loss cylinder with $q_{\text {prior }}>q_{l c}>q_{o b s}$,

$$
\mathbf{H}_{\mathrm{obs}}=\mathbf{H}_{\text {prior }}+\Delta \mathbf{H}_{\text {tide }}
$$

We recast this as

$$
q_{\text {prior }} \approx q_{\text {obs }}+\frac{\Delta \mathbf{H}_{\text {tide }}{ }^{2}}{2 \mathrm{GM}_{\odot}}-\frac{\Delta \mathbf{H}_{\text {tide }} \cdot \mathbf{H}_{\text {obs }}}{\mathrm{GM}_{\odot}} .
$$

from which it is clear that if weak tidal perturbations dominate in making Oort cloud comets observable, the tidal characteristic,

$$
S \equiv \operatorname{Sign}\left(\Delta \mathbf{H}_{\text {tide }} \cdot \mathbf{H}_{\text {obs }}\right)=\operatorname{Sign}(-\sin B \cos \alpha(1+\epsilon \cos 2 L)-\epsilon \sin \alpha \sin 2 L),
$$

will more often be -1 than +1 . Here $B, L$ and $\alpha$ are the observed values of orbital angles. Modeling results (Matese and Lissauer 2002) confirm this implication. Fouchard (2003) has developed an iterative mapping model which may prove to moderate the small errors associated with the zero-order mapping represented by Eq. 4 .

A graphical illustration of this theme in Fig. 1 shows the phase space changes in $\mathbf{H}$ for a specific choice of $\widehat{Q}$ and $a$ over the course of a single orbit period $P_{a}$. As comets recede from the planetary region on their prior orbits, the interior of the loss cylinder phase space region is emptied of new comets by planetary perturbations. The exterior region remains uniformly populated in the standard model. To lowest order in $q / a$, i.e., the near-parabolic phase space region just outside the loss cylinder boundary, the vector displacement in specific angular momentum, $\boldsymbol{\Delta} \mathbf{H}_{\text {tide }}$, given in Eq. 2, is independent of the prior value of angular momentum, $\mathbf{H}_{\text {prior }}$, and depends only on $a$ and the major axis orientation, $\widehat{Q}$, which are taken to be fixed for the phase space of Fig. 1. In a single orbit all nearby phase space points, filled and empty, are displaced uniformly.

The magnitude of the single-orbit displacement is strongly dependent on $a$, varying as $a^{7 / 2}$. Small- $a$ comets are defined here as having negligible perturbations, unable to repopulate the observable zone where comets become discernable, i.e., inner Oort cloud comets. Large- $a$ comets are defined here as those having strong tidal perturbations resulting in large displacements in $\mathbf{H}$ that completely refill the observable zone, 
making $S=\mp 1$ equally likely. Intermediate- $a$ comets are defined here as those that are weakly perturbed and only partially refill the observable zone. Intermediate- $a$ comets preferentially have $S=-1$, as seen in Fig. 1. In this context intermediate- $a$ new comets have the smallest observed values of $a$ among the new comets. The boundaries between small- $a$, intermediate- $a$ and large- $a$ depend weakly on $B$ and $L$, as can be inferred from Eq. 2. Finally, we see that intermediate- $a$ comets preferentially have large $q$ since they "just barely" enter the observable zone.

Planetary impulses that empty the loss cylinder primarily change comet energies and not angular momenta. Those comets that are perturbed to the inner Oort cloud will have nearly unchanged values of all orbital elements excepting $a$ when they return as young or old comets. Therefore, if young and old comet generations are indeed dominated by daughters of the new family (Levison et al. 2001) they should also have a preponderance of values of $S=-1$. No correlation of $S=-1$ with $a$ for these generations is expected, of course. But the correlation of $S=-1$ with large $q$ should remain for these generations.

Therefore, independent of the in situ distribution in $a$, if the galactic tidal interaction with the Oort cloud dominates stellar impulses in producing long period comets we should see

- a preponderance of long period comets with $S=-1$ over those with $S=+1$,

- a correlation indicating that long period comets with large observed values of $q$ will show this preponderance more strongly than comets with small $q$, and

- a further correlation for new comets in which $S=-1$ and large $q$ correlate with small observed values of $a$.

Conversely, if the galactic tide does not dominate stellar impulses in making long period comets observable at the present epoch, the unique tidal characteristic $S$ should be a random variable and should be uncorrelated with $q$ and $a$. 


\section{OBSERVED ORBITAL ELEMENT DISTRIBUTIONS}

Our data are taken from the 14th Catalogue of Cometary Orbits (Marsden and Williams 2002), from which we convert the ecliptic Eulerian orbital angles into the galactic angles $B, L$ and $\alpha$. The data contain 142 new comets, 87 young comets and 156 old comets of all quality classes. The quality class predominantly distinguishes the accuracy of the original semimajor axis determination. We include all 385 comets since the accuracy of all other orbital elements is more than sufficient for all analyses not involving $a$.

\subsection{Distribution in Latitude}

The first observational indication that the galactic tide dominates involved the distribution in the galactic latitude of aphelion, $B$ (Delsemme 1987). In Eq. 2, we see that the dominant disk tidal term in the perturbation is $\propto \sin B \cos B$. The latitude $B$ is sensibly constant in the course of a single orbit. Therefore, if the tide dominates we should expect deficiencies of major axis orientations along the galactic poles and the galactic equator, and a peak near $B=45^{\circ}$. One might argue that observational selection effects can artificially produce an equatorial gap, but any polar gap will be more difficult to attribute to an observational selection effect.

In Fig. 2 we show the results for old, young and new comet populations. The data are presented as distributions in $\sin B$, which would be uniform for a random distribution. Polar and equatorial gaps are clear in the new family, but are marginal for the young and old generations. Further, the observed

peaks are all slightly removed from the predicted peaks which occur at $\sin B \approx \pm 0.7$ when a uniform prior distribution in $\sin B$ is assumed (Matese and Lissauer 2002).

A hypothesis that the observed young + old latitude distributions are samples taken from the same population as the observed new distribution can be readily rejected at a high confidence level by performing a $\chi^{2}$ test on the binned distributions. In itself this does not falsify the supposition that planetary processing of new Oort cloud comets accounts for the majority of young and old comets since planetary perturbations 
depend on orbit orientation. So we look to the tidal characteristic $S$ to further investigate this question.

\subsection{Distribution in the Tidal Characteristic $S$}

In Fig. 3 we show the distributions of $S$ as a function of $q$ for each generation of comets. A simple statistical analysis that one can make is to test the null hypothesis that these distributions are samples of a random distribution with $S=\mp 1$ equally likely, as would occur if the galactic tide was a negligible contributor to the comet flux and stellar impulses dominated. The binomial probabilities that we would get

as many (or more) values of $S=-1$ for these generations are $p=3 \times 10^{-8}$ for all 385 comets, $p=8 \times 10^{-6}$ for 142 new comets and $p=3 \times 10^{-4}$ for 243 young + old comets. This is strong evidence that the galactic tide dominates in making new comets observable, and that young + old comets are predominantly daughters of the new family.

The ratio of the number of comets with $S=\mp 1$ is 2.2 for the new family and 1.6 for the young + old generations. The reduction of this ratio may be due to a modest fraction of the young + old population being daughters of the Kuiper belt/scattered disk. Additionally/alternatively, the ratio may be reduced as comets age by fading (Wiegert and Tremaine 1999), including the mass disruption of comets (Levison et al. 2002). One sees in Fig. 3 that the new family has proportionally more large- $q$ ( $>2$ AU) members than the young + old generations. As occurred for the latitude distributions, one can reject, at a high confidence level, the hypothesis that the new distribution in $q$ is taken from the same population as the young + old $q$ distribution. Matese and Lissauer (2002) have shown theoretically that $S=-1$ correlates with large $q$ for new Oort cloud comets. This is visually suggested in Fig. 3 and will be further analyzed in the next subsection. Since fading makes large- $q$ comets comparatively more difficult to observe on successive orbits, this can explain the relative deficiency of $S=-1$ comets from the young + old generations. Models of the galactic tidal interaction with the Oort cloud (Matese and Lissauer 2002) predict a modest increase in the $q$ distribution with increasing values of $q$. If the $q<2 \mathrm{AU}$ observations are taken to be complete for new comets, we conclude that $\approx 1 / 2$ of the new comets interior to $5 \mathrm{AU}$ are observed. 


\subsection{Correlation Between $q$ and $S$}

The preponderance of the tidal characteristic $S=-1$ over $S=+1$ cannot, in itself, be taken as evidence for the dominance of the galactic tide. We must self-consistently demonstrate that the explanation suggested for this, illustrated in Fig. 1, is observationally supported. Independent of the excess of values $S=-1$, does $S=-1$ correlate with larger values of $q$ ? A lack of correlation, or a correlation with smaller values of $q$, would indicate that the dynamical arguments presented here must be rejected. A statistical measure of this association can be obtained from the Kendall rank correlation test (Mathematica 1999). Considering the complete set of data, we find the probability that we would get as strong a correlation if $q$ and $S$ were unassociated (the null hypothesis) is $p(q S)=0.0012$. We can reject the null hypothesis at the level of $\approx 3 \sigma$. The correlation holds for both the new family $(2.1 \sigma)$ and the young + old generations $(1.9 \sigma)$.

\subsection{Correlation Between $a$ and $S$}

We now investigate the prediction that $S=\mp 1$ should be equally likely for large- $a$ new comets, and that there should be a preponderance of $S=-1$ for intermediate- $a$ new comets (see Fig. 1). In considering the $a-S$ correlation, we restrict ourselves to the highest quality class (Marsden et al. 1978) of new comets, since an accurate determination of the original semimajor axis is crucial here. There are 71 new comets of this class, $1 \mathrm{~A}$. In terms of the original orbital energy parameter $x \equiv 10^{6} \mathrm{AU} / a$, class $1 \mathrm{~A}$ new comets have a mean formal error of 5 units (Kresák 1992), but the uncertainty due to unmodeled outgassing effects is likely to be somewhat larger (Whitmire and Matese 2003).

Figure 4 shows the distribution in a for class 1A new comets. Separately displayed in each histogram bin are the contributions of the tidal characteristic $S=-1$ and $S=+1$. There are 49 values of $S=-1$ and 22 values of $S=+1$ in this family. We see that there are roughly equal numbers of $S=\mp 1$ for $x \leq 30$. The inference is that the refilling of the observable zone of the loss cylinder by the galactic tide is near-complete for semimajor axes $a \geq 33,000$ AU. We find a preponderance of $S=-1$ for new comets with $30<x<100$. 
The probability that we would get as much correlation between $S=-1$ and small $a$ if in fact the variables were unassociated (the null hypothesis here) is $p(a S)=0.0018$. As before, we can reject the null hypothesis at the level of $\approx 3 \sigma$, but cannot reject the hypothesis that the observations are a sample of a population in which the galactic tide dominates.

Fernandez (2002) has conjectured that the $x \leq 30$ portion of the outer Oort cloud "remembers" its primordial orbital angular momentum since 21 of these 30 comets have ecliptic prograde orbits. But this is the energy range experiencing the largest changes in angular momentum magnitude and orientation angle,

$\alpha$, during the prior orbit and the prior orbital angular momentum distribution must be determined to see if the conjecture can be sustained. He also finds that comets with $30<x<100$ are preferentially polarized in the ecliptic retrograde sense, reversing the polarization for large- $a$ comets. His theme is that retrograde comets effectively have a smaller loss cylinder radius than prograde comets since planetary impulses at the same $q_{\text {prior }}$ will be, on average, smaller for the larger relative velocities associated with retrograde orbits. The smaller loss cylinder radius for retrograde comets then means that for these comets the distinctions between large- $a$, intermediate- $a$ and small- $a$, discussed in Fig. 1, can be thought of as shifting slightly toward the higher-flux inner Oort cloud. For 1A comets in this energy range 25 of 41 comets have ecliptic retrograde orbits. We see evidence for this plausible notion, but not at a statistically significant level. In any case, the arguments presented above do not depend on the in situ Oort cloud distribution being unpolarized.

\section{SUMMARY AND CONCLUSIONS}

We have described how the dynamics of the galactic tidal interaction predicts specific properties for a combination of angular orbital elements,

$$
S \equiv \operatorname{Sign}\left(\Delta \mathbf{H}_{\text {tide }} \cdot \mathbf{H}_{\text {obs }}\right)=\operatorname{Sign}(-\sin B \cos \alpha(1+\epsilon \cos 2 L)-\epsilon \sin \alpha \sin 2 L)
$$

If the galactic tide indeed dominates in making Oort cloud comets observable, then we should see: 
- a preponderance of new comets with $S=-1$ over those with $S=+1$,

- new comets with the largest observed values of $a$ equally likely to have $S=\mp 1$, while those with the smallest observed values of $a$ should preferentially have $S=-1$, and

- a three-fold correlation between new comet orbital elements $S=-1$, small observed values of $a$, and large observed values of $q$.

Further, if young and old comets are predominantly daughters of new comets we should similarly find:

- a preponderance of young and old comets with $S=-1$ over those with $S=+1$, and

- young and old comets with large $q$ will show this preponderance more strongly than those with small q, i.e., a two-fold correlation between $S=-1$ and large $q$.

All of these subtle predictions are found to be manifest in the observational data at statistically significant levels, much stronger than have been inferred previously from distributions of major axis latitudes.

We conclude that the data provide convincing evidence of this unique imprint of galactic tidal dominance in making new Oort cloud comets observable. A further conclusion is that young and old comets are indeed predominantly daughters of the new comet population. Finally, we find the data to be of sufficiently high quality and sufficiently free of observational bias to search for secondary impulsive contributions to the Oort cloud comet flux.

\section{ACKNOWLEDGMENTS}

The authors thank J. Fernandez for a constructive review. JJL received support from Planetary Geology and Geophysics Grant 344-30-50-01. JJM acknowledges discussions with D. P. Whitmire. 


\section{REFERENCES}

Delsemme, A. H. 1987. Galactic tides affect the Oort cloud: an observational confirmation. Astron. Astrophys. 187, 913-918.

Fernandez, J. 1981. New and evolved comets in the Solar System. Astron. Astrophys. 96, 26-35.

Fernandez, J. 2002. Changes in the inclination-distribution of long-period comets with the orbital energy. Proc. of ACM2002 (ESA-SP-500), 303-304.

Fouchard, M. 2003. New fast models of the galactic tide. Mon. Not. R. Astron. Soc. 349, 347-356.

Heisler, J., and S. Tremaine 1986. Influence of the galactic tidal field on the Oort cloud. Icarus 65, 13-26.

Holmberg, J., and C. Flynn 2000. The local density of matter mapped by HIPPARCOS. Mon. Not. R. Astron. Soc. 313, 209-216.

Kresák, L. 1992. Are there any comets coming from interstellar space? Astron. Astrophys. 259, 682-691.

Levison, H. F., L. Dones, and M. J. Duncan 2001. The origin of Halley-type comets: Probing the inner Oort cloud. Astron. J. 121, 2253-2267.

Levison, H. F., A. Moribidelli, L. Dones, R. Jedicke, P. A. Wiegert and and W. F. Bottke, Jr. 2002. The mass disruption of Oort cloud comets. Science 296, 2212-2215.

Marsden, B. G., Z. Sekanina, and E. Everhart 1978. New Osculating orbits for 110 comets and analysis of original orbits for 200 comets. Astron. J. 83, 64-71.

Marsden, B. G., and G. V. Williams 2002. Catalogue of Cometary Orbits, 14th Ed. Smithsonian Astrophysical Observatory, Cambridge, MA.

Matese, J. J., and P. G. Whitman 1992. A model of the galactic tidal interaction with the Oort comet cloud. Cel. Mech. Dyn. Astron. 54, 13-36. 
Matese, J. J., and D. P. Whitmire 1996. Tidal imprint of distant galactic matter on the Oort comet cloud. Ap. J. 472, L41-L43.

Matese, J. J., and J. J. Lissauer 2002. Characteristics and frequency of weak stellar impulses of the Oort cloud. Icarus 157, 228-240.

Merrifield, M. R. 1992. The rotation curve of the Milky Way to $2.5 R_{o}$ from the thickness of the HI layer. Astron. J. 103, 1552-1563.

Oort, J. H. 1950. The structure of the cloud of comets surrounding the Solar System, and a hypothesis concerning its structure. Bull. Astron. Inst. Neth. 11, 91-110.

Whitmire, D. P., and J. J. Matese, 2003. The Pioneer 10 anomalous acceleration and Oort cloud comets. Icarus 165, 219-222.

Wiegert, P., and S. Tremaine 1999. The evolution of long-period comets. Icarus 137, 84-122.

Wolfram Research 1999. Mathematica, 4th Ed. Cambridge University Press.

This preprint was prepared with the AAS LATEX macros v4.0. 


\section{Specific Angular Momentum Plane, $H$}

\section{Refilled portion of loss cylinder}

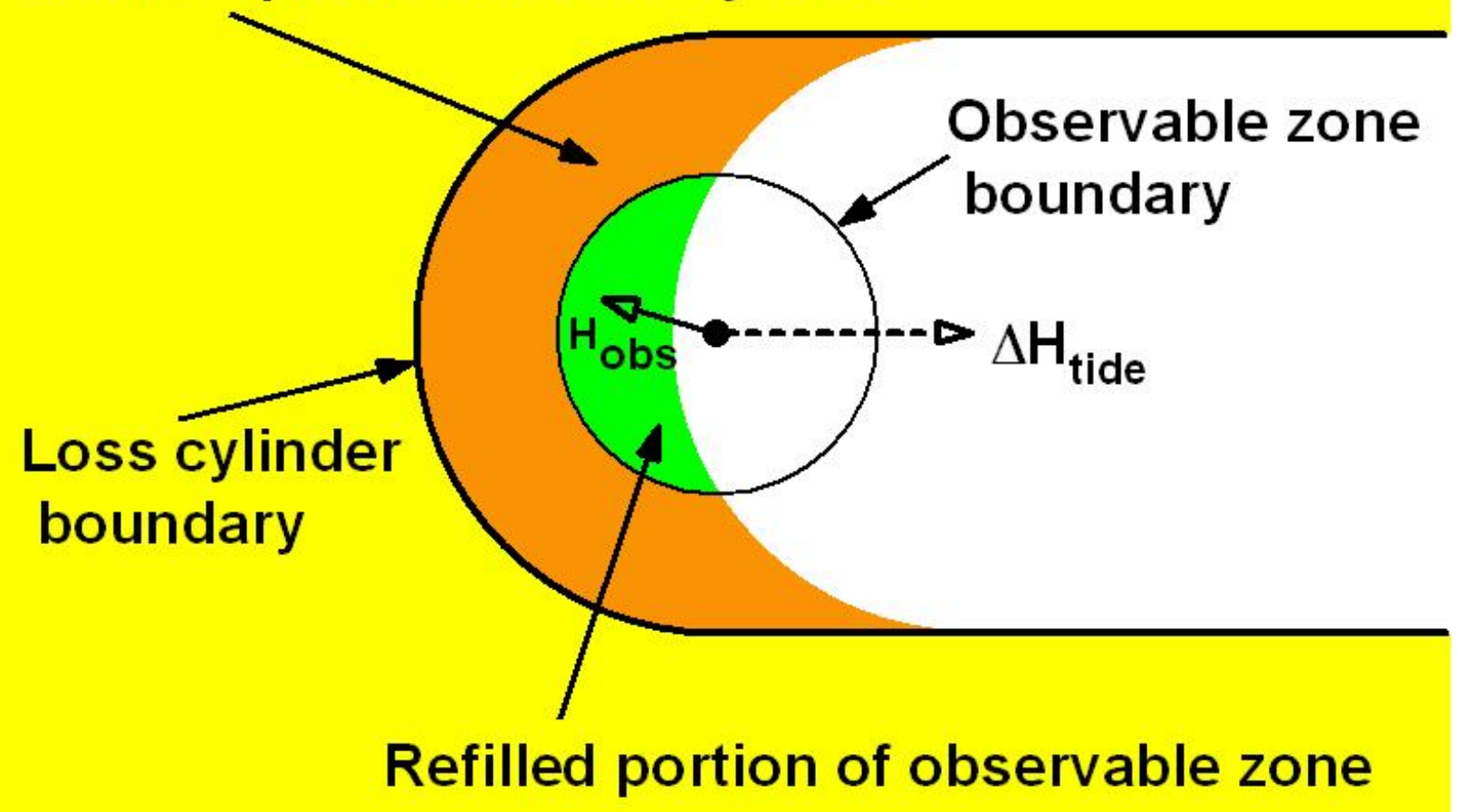

Fig. 1.- Schematic illustration of the specific angular momentum phase space of comets for a specified $a$ and $\widehat{Q}$, the plane's normal direction. Yellow shaded region outside the loss cylinder boundary $\left(H>H_{l c} \propto \sqrt{q_{l c}}\right)$ denotes the filled phase space of comets leaving the planetary region on their prior orbits. The uniform displacement of all phase space points one orbit later by a tidal perturbation $\Delta \mathbf{H}_{\text {tide }}$ is shown. The observable zone $\left(H_{o b s} \propto \sqrt{q_{o b s}}\right)$ is indicated. It remains unfilled for negligible perturbations of small- $a$ comets, becomes totally refilled for strong perturbations of large- $a$ comets, and, as illustrated here, is partially refilled (green shaded region) for weak perturbations of intermediate- $a$ comets. The tidal characteristic $S \equiv \operatorname{Sign}\left(\mathbf{H}_{\text {obs }} \cdot \Delta \mathbf{H}_{\text {tide }}\right)=-1$ for the refilled portion of the observable phase space here. Over successive orbits the loss cylinder shadow region of unfilled phase space (shaded white) will form. 


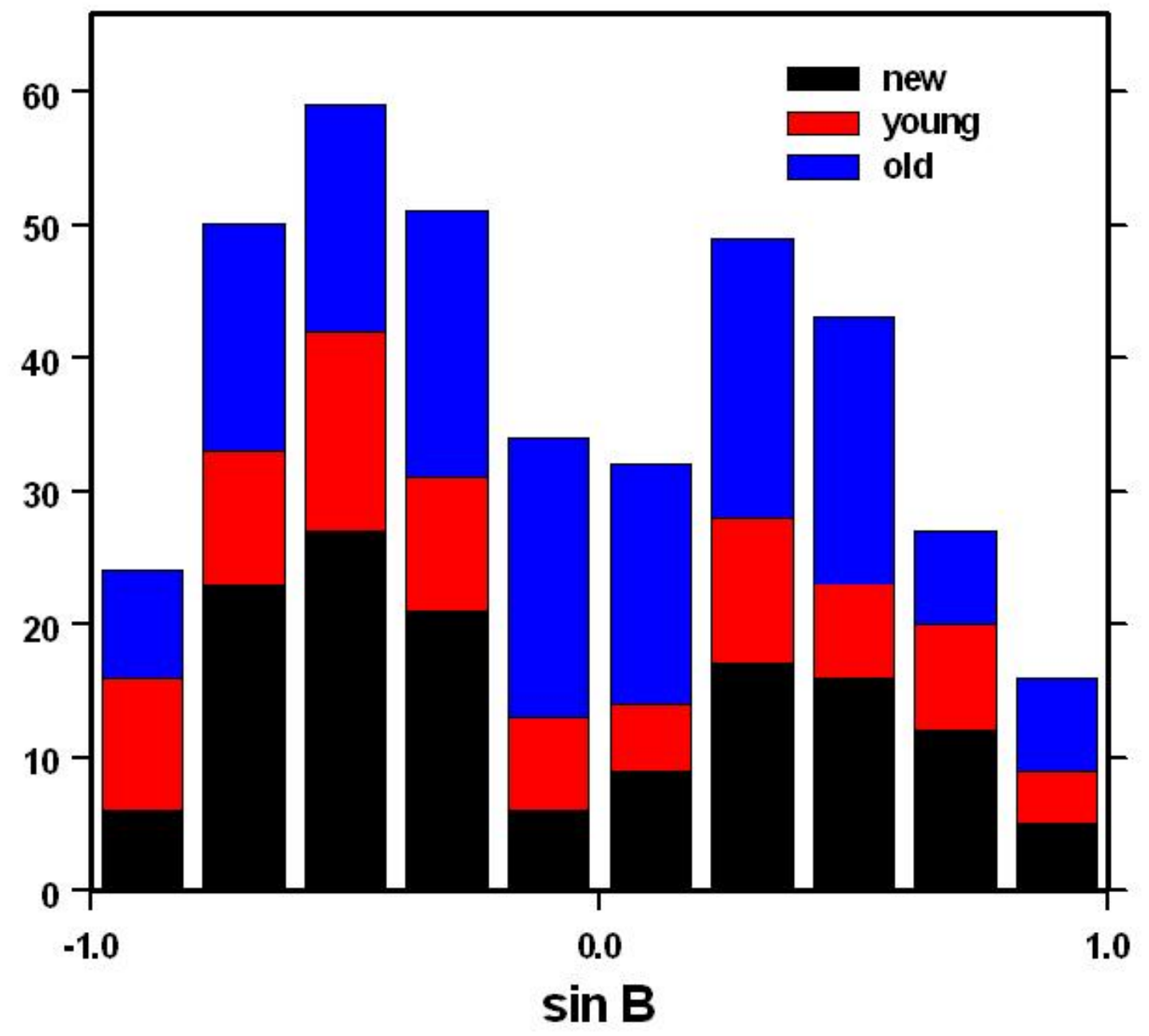

Fig. 2.- The $\sin B$ distributions for each generation. 


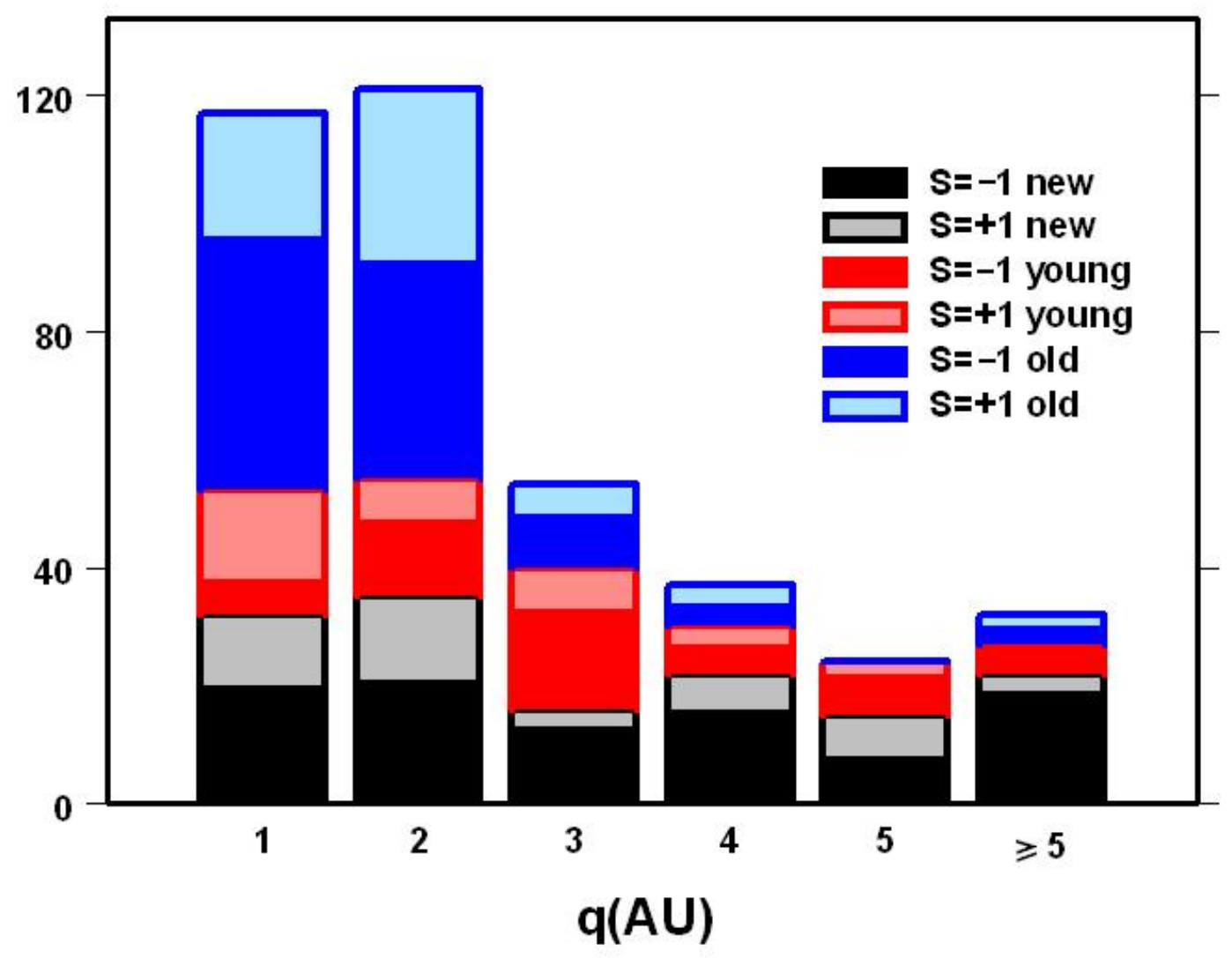

Fig. 3.- The $q-S$ distributions for each generation. Results are binned in 1 AU intervals for $q<5$ AU. The ratios of $S=-1$ to $S=+1$ for each generation are; new (97-/45+), young (53-/34+) and old (96-/60+). 


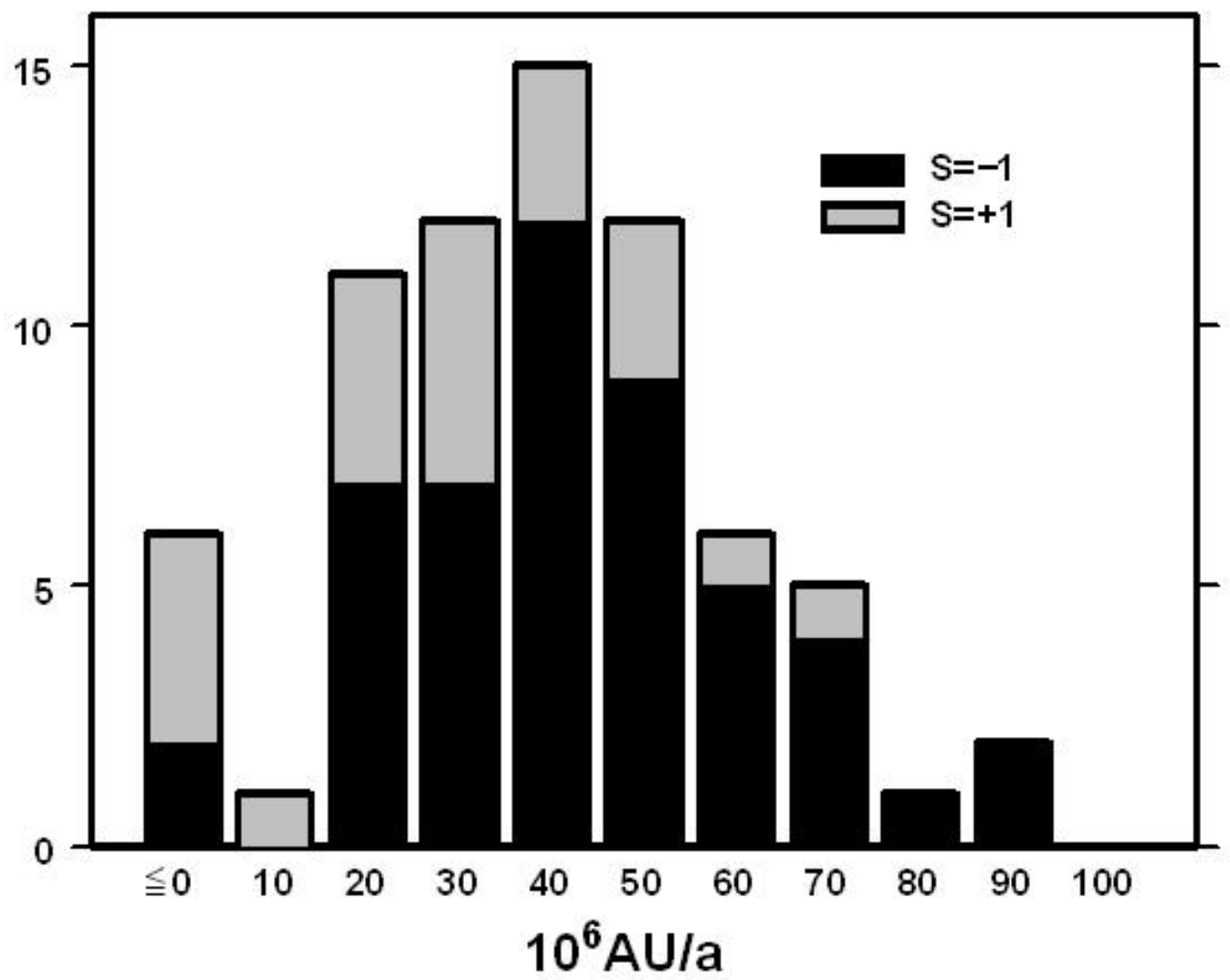

Fig. 4.- The $a-S$ distribution for class 1 A new comets. 